\title{
Global sulfur dioxide emissions and the driving forces
}

Qirui Zhong ${ }^{\dagger}$, Huizhong Shen ${ }^{*}$, Xiao Yun ${ }^{\dagger}$, Yilin Chen ${ }^{\dagger *}$, Yu'ang Ren ${ }^{\dagger}$, Haoran Xu^, Guofeng Shen ${ }^{\dagger}$, Wei Du${ }^{\dagger}$, Jing Meng", Wei $\mathrm{Li}^{\dagger}$, Jianmin $\mathrm{Ma}^{\dagger}$, Shu Tao ${ }^{\dagger}$

$\uparrow$ College of Urban and Environmental Sciences, Laboratory for Earth Surface Processes, Sino-French Institute for Earth System Science, Peking University, Beijing 100871, China

+ School of Civil and Environmental Engineering, Georgia Institute of Technology, Atlanta 30318, USA

$\S$ Key Laboratory of Geographic Information Science of the Ministry of Education, School of Geographic Sciences, East China Normal University, Shanghai 200241, China

\| The Bartlett School of Construction and Project Management, University College London, London WC1E 7HB, UK

* Corresponding author: hshen73@gatech.edu

${ }^{1}$ Present address: School of Civil and Environmental Engineering, Georgia Institute of Technology, Atlanta 30318, USA

There are 6 figures and 6 tables. 

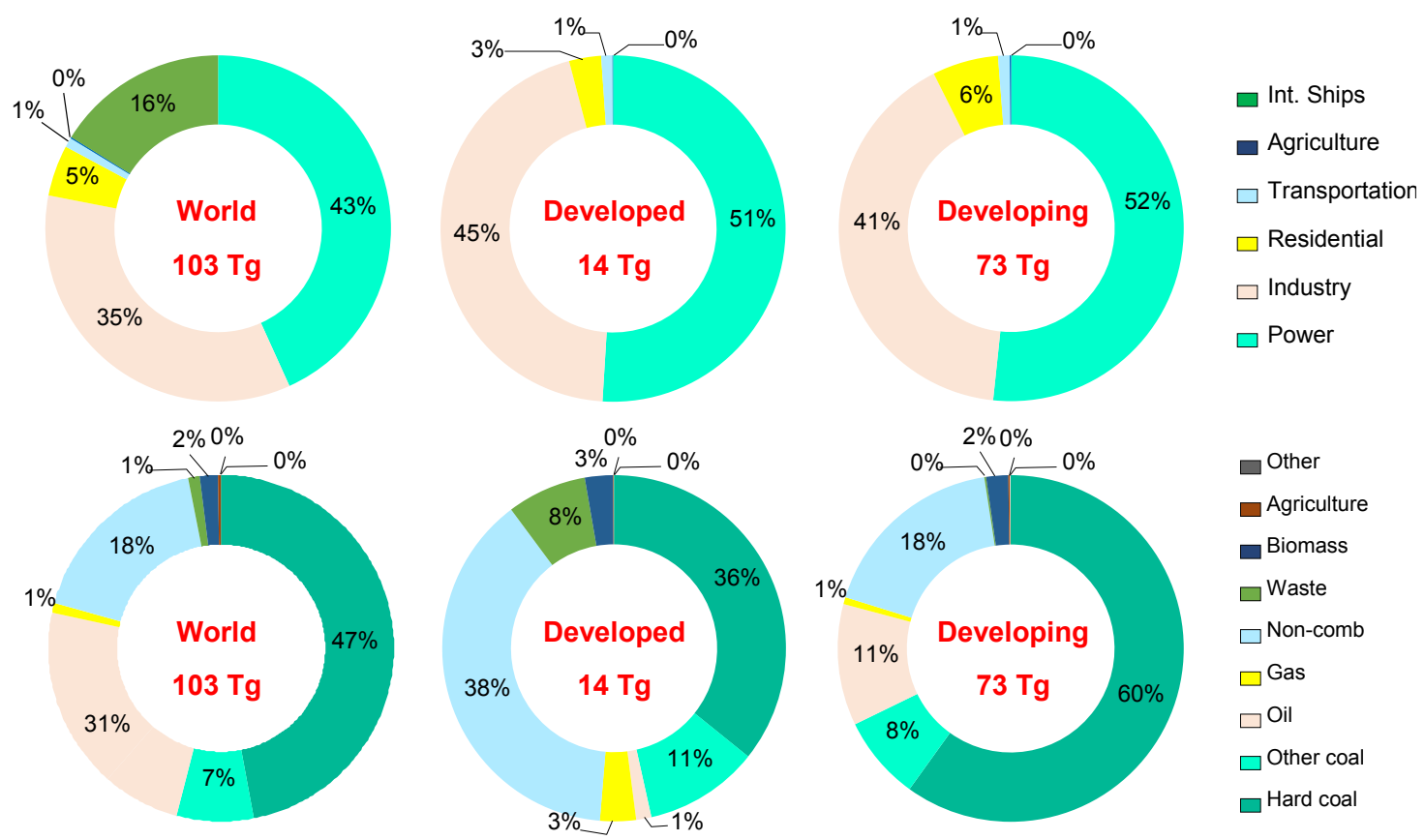

Fig. S1 Source profiles of anthropogenic $\mathrm{SO}_{2}$ emissions for world, developed, and developing countries in view of emission sector (top panel) and fuel type (bottom panel). The centered numbers show anthropogenic $\mathrm{SO}_{2}$ emissions in 2014. Note that emissions from international shipping was only considered in global total emissions. Therefore, the global total emission was higher than the sum of the emissions from the developed and developing countries, and the global contributions of certain fuel types such as noncombustion sources may not fall in between of the contributions for developed and developing countries.
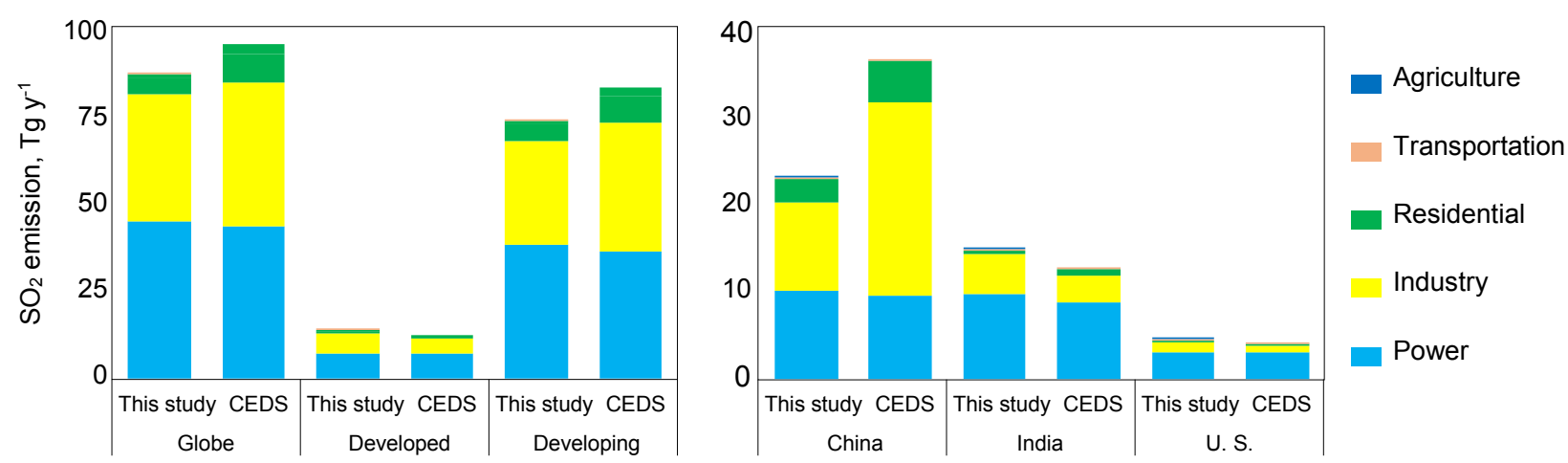

Fig. S2 Comparison of $\mathrm{SO}_{2}$ emission source profiles between CEDS and this study for globe and typical regions. 


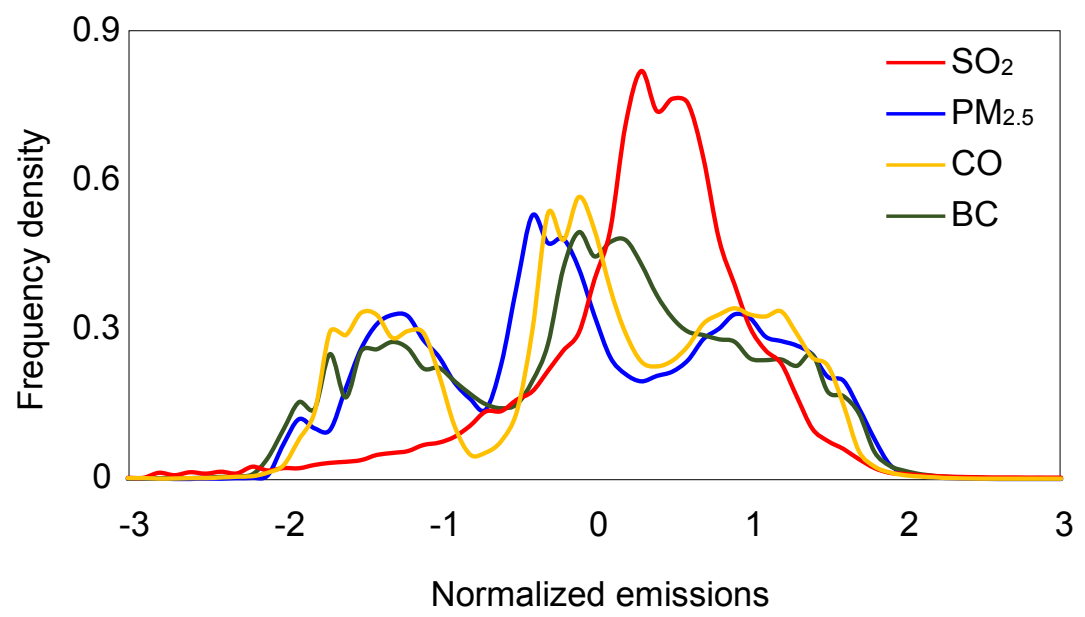

Fig. S3 Frequency distribution of gridded $\mathrm{SO}_{2}$ emissions and emissions for incomplete combustion pollutants (i.e., $\mathrm{CO}, \mathrm{PM}_{2.5}, \mathrm{BC}$ ).
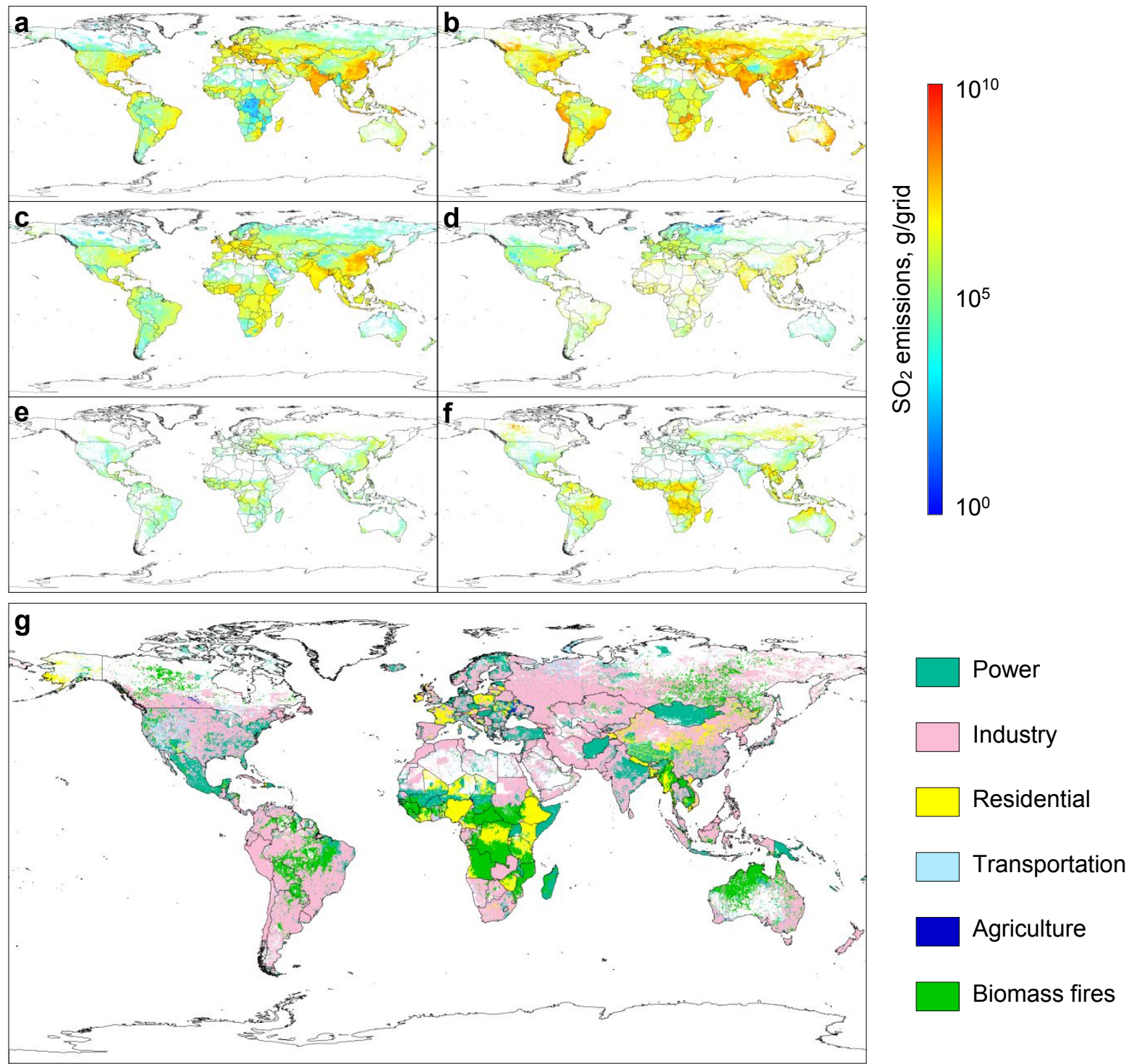

Fig. S4 Spatial distribution of $\mathrm{SO}_{2}$ emissions from power generation (a), industry (b), residential sector (c), transportation (d), agriculture (e), and biomass fires (f). The sector with predominant contribution on grid level is illustrated in $\mathrm{g}$. 

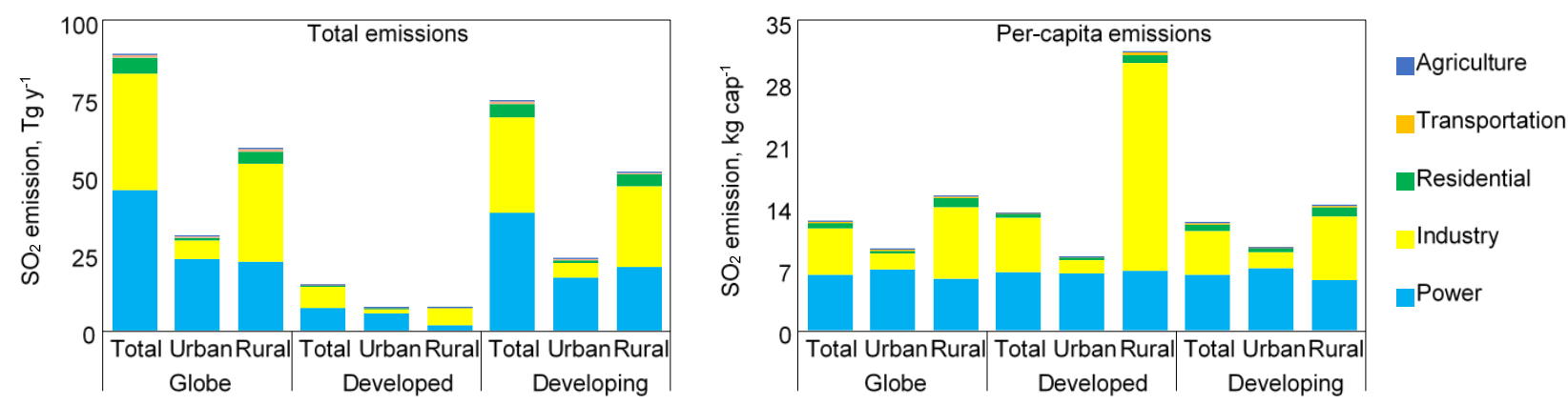

Fig. S5 Source profile of total and per-capita $\mathrm{SO}_{2}$ emissions for globe, developed, and developing countries.
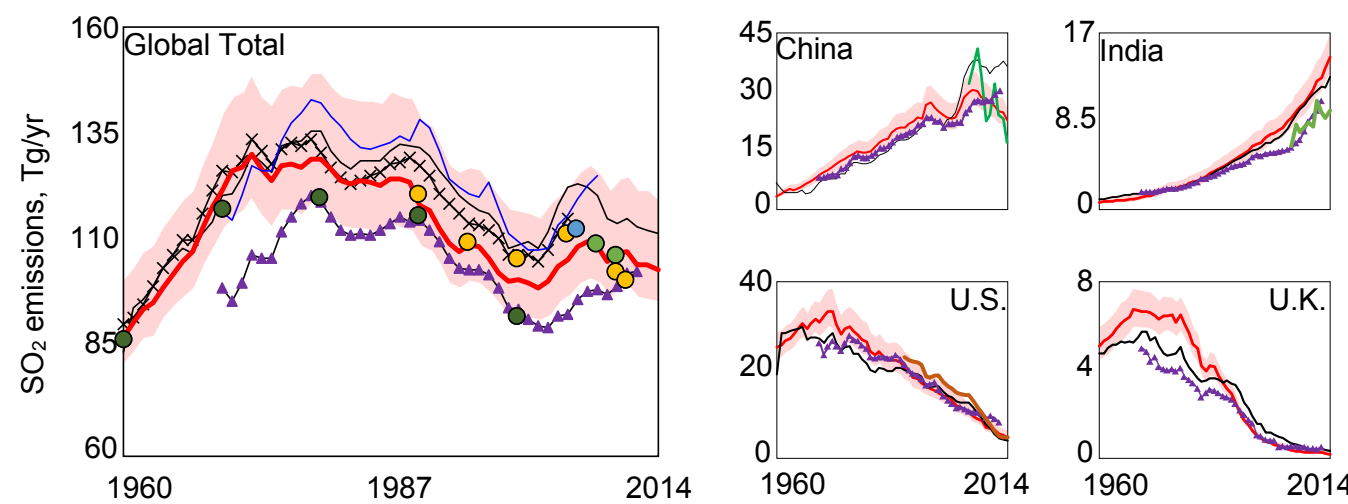

- HTAP2.2

- Klimont, et al., 2013

- Lamarque et al., $201 \mathrm{c}$

- Lee et al., 2011

_ـEDGAR4.3.2
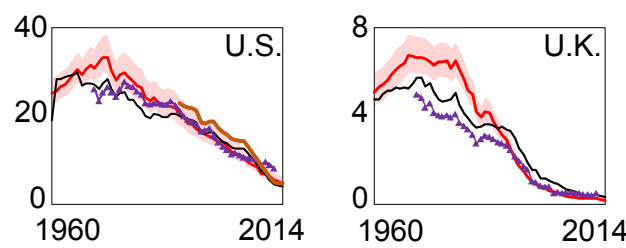

- Smith11

_ CEDS

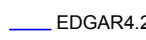

This study

__ Li et al., 2017

EPA-NE

Fig. S6 Comparisons of anthropogenic $\mathrm{SO}_{2}$ emission trends between this study and previous literatures for globe and typical countries. 

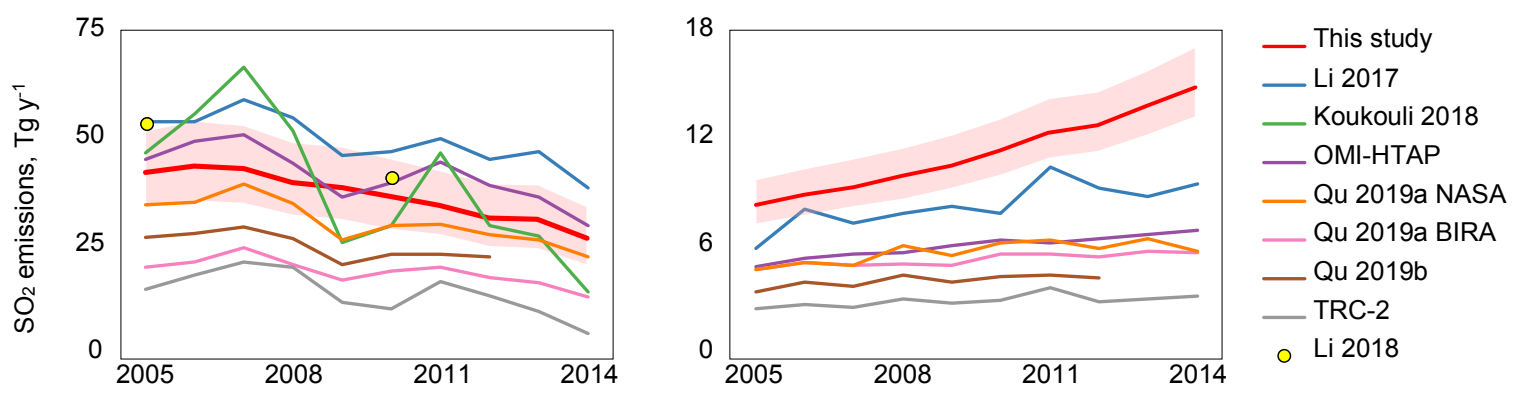

Fig. S7 Comparisons of the annual total $\mathrm{SO}_{2}$ emissions in China and India with those estimated from topdown studies ${ }^{1-7}$. The red shaded area shows the interquartile range of emissions estimated in this study.
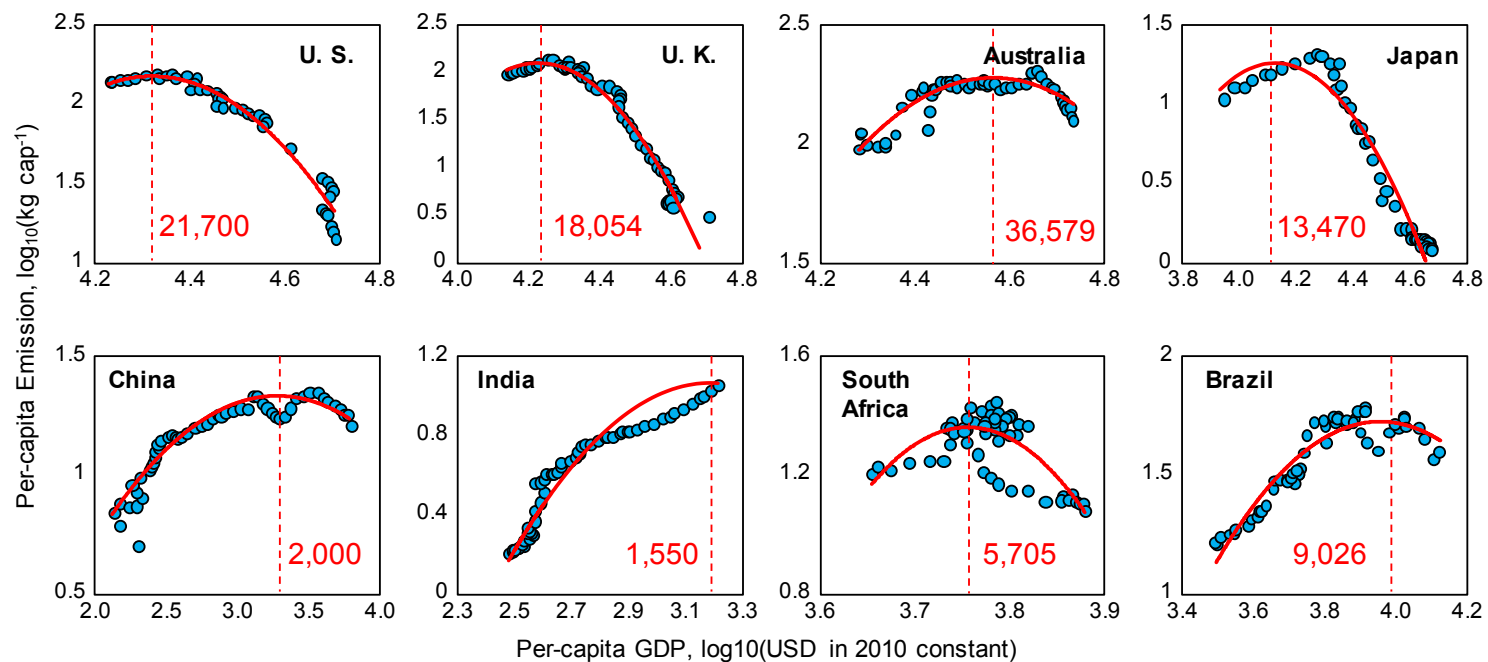

Fig. S8 Historical trajectories of per-capita $\mathrm{SO}_{2}$ emissions as dependence on per-capita GDP for selected counties. The solid lines and dashed lines show the empirical quadratic regression (EKC) and the corresponding turning point with exact GDP values labeled. The top and bottom panels typically indicate developed and developing counties, respectively. 
Table S1 Comparisons of bottom-up $\mathrm{SO}_{2}$ emissions from previous literatures and local governments in 2010.

\begin{tabular}{ccl}
\hline Region/Country & $\mathbf{S O}_{2}$ emissions, $\mathbf{T g}^{a}$ & Data source \\
\hline U. S. & 10.11 & EDGAR_v4.3 $^{8}$ \\
& 6.71 & CEDS $^{9}$ \\
& 7.18 & ECLIPSE $^{10}$ \\
& 7.70 & 11 \\
\hline U. K. & 7.66 & US-EPA $^{12}$ \\
\hline China & 0.446 & EDGAR_v4.3 $^{8}$ \\
& 0.342 & CEDS $^{9}$ \\
& 0.397 & ECLIPSE $^{10}$ \\
& 0.449 & LRTAP $^{13}$ \\
\hline India & 30.0 & EDGAR_v4.3 $^{8}$ \\
& 34.7 & CEDS $^{9}$ \\
& 35.0 & ECLIPSE $^{10}$ \\
& 27.8 & 14 \\
\hline & 21.9 & 15 \\
\hline
\end{tabular}

a. Only anthropogenic emissions were considered. 
Table S2 List of geomean and standard deviation of EFs (originally in $\mathrm{g} / \mathrm{kg}$ ) collected from literature and database.

\begin{tabular}{|c|c|c|c|}
\hline Sector & Sources & EFs (geomean \pm SD) & Ref. \\
\hline \multirow{13}{*}{$\begin{array}{l}\text { Power } \\
\text { generation }\end{array}$} & Anthracite & $a$ & 17 \\
\hline & Coking coal & $a$ & 17 \\
\hline & Bituminous coal & $a$ & 17 \\
\hline & Lignite & $\boldsymbol{b}$ & 17 \\
\hline & Peat & $\boldsymbol{b}$ & 17 \\
\hline & Gas/diesel & $c$ & 17 \\
\hline & Residue fuel oil & $c$ & 17 \\
\hline & Solid biomass & $0.66 \pm 1.74$ & 18 \\
\hline & Biogas & $0.15 \pm 0.03$ & 18 \\
\hline & Municipal waste & $5.66 \pm 3.22$ & 18 \\
\hline & Industrial waste & $5.66 \pm 3.22$ & 18 \\
\hline & Dry natural gas & $0.15 \pm 0.03$ & 18 \\
\hline & Natural gas liquid & $0.15 \pm 0.03$ & 18 \\
\hline \multirow{15}{*}{$\begin{array}{l}\text { Industry } \\
\text { combustion }\end{array}$} & Anthracite & $a$ & 17 \\
\hline & Coking coal & $a$ & 17 \\
\hline & Bituminous coal & $a$ & 17 \\
\hline & Lignite & $\boldsymbol{b}$ & 17 \\
\hline & Peat & $\boldsymbol{b}$ & 17 \\
\hline & Residue fuel oil & $c$ & 17 \\
\hline & Gas/diesel & $c$ & 17 \\
\hline & Crude oil refinery & $c$ & 17 \\
\hline & Biogas & $0.15 \pm 0.03$ & 18 \\
\hline & Municipal waste & $5.66 \pm 3.22$ & 18 \\
\hline & Industrial waste & $5.66 \pm 3.22$ & 18 \\
\hline & Solid biomass & $0.66 \pm 1.74$ & 18 \\
\hline & Dry natural gas & $0.15 \pm 0.03$ & 18 \\
\hline & Natural gas liquid & $0.15 \pm 0.03$ & 18 \\
\hline & Natural gas flaring & $0.15 \pm 0.03$ & 18 \\
\hline \multirow{5}{*}{$\begin{array}{l}\text { Non-combustion } \\
\text { industry }{ }^{d}\end{array}$} & Copper smelting & 1060 & 19 \\
\hline & Lead smelting & 490 & 19 \\
\hline & Zinc smelting & 1000 & 19 \\
\hline & Nickel smelting & 150 & 19 \\
\hline & Natural gas production & 0.15 & 19 \\
\hline \multirow{19}{*}{$\begin{array}{l}\text { Residential } \\
\text { sector }\end{array}$} & Solid waste & $0.66 \pm 1.74$ & $18,20-23$ \\
\hline & Anthracite used & $14.4 \pm 1.47$ & 18 \\
\hline & Coking coal & $14.4 \pm 1.47$ & 18 \\
\hline & Bituminous & $14.4 \pm 1.47$ & 18 \\
\hline & Lignite & $14.4 \pm 1.47$ & 18 \\
\hline & Peat & $14.4 \pm 1.47$ & 18 \\
\hline & Coal briquettes & $14.4 \pm 1.47$ & 18 \\
\hline & Patent fuel & $14.4 \pm 1.47$ & 18 \\
\hline & Gasoline/diesel & $0.65 \pm 0.63$ & 24 \\
\hline & Fuel oil & $0.65 \pm 0.63$ & 24 \\
\hline & Gas works gas & $0.65 \pm 0.63$ & 24 \\
\hline & Coke oven gas & $0.65 \pm 0.63$ & 24 \\
\hline & Charcoal & $0.65 \pm 0.63$ & 24 \\
\hline & Corncob & $0.31 \pm 1.49$ & 18 \\
\hline & Brush wood & $0.65 \pm 0.63$ & $18,20-22,25-27$ \\
\hline & Liquid petroleum gas & $0.15 \pm 0.03$ & 24 \\
\hline & Dry natural gas & $0.65 \pm 0.63$ & 24 \\
\hline & Natural gas liquid & $0.65 \pm 0.63$ & 24 \\
\hline & Kerosene & $0.65 \pm 0.63$ & 24 \\
\hline
\end{tabular}




\begin{tabular}{|c|c|c|c|}
\hline & Biogas & $0.65 \pm 0.63$ & 24 \\
\hline & Firewood & $0.65 \pm 2.09$ & $18,20-22,25-27$ \\
\hline & Straw & $0.31 \pm 1.49$ & $18,20,23,5-27$ \\
\hline & Dung cake & $2.16 \pm 2.73$ & $18,20-22,25$ \\
\hline \multirow[t]{5}{*}{ Transportation } & Motor vehicle gasoline & $e$ & See Table S4 \\
\hline & Motor vehicle diesel & $\boldsymbol{e}$ & See Table S4 \\
\hline & Biodiesel & $e$ & See Table S4 \\
\hline & Jet kerosene & $2.5 \pm 0.63$ & 18 \\
\hline & Aviation gasoline & $2.5 \pm 0.63$ & 18 \\
\hline \multirow[t]{2}{*}{ Agriculture } & Agriculture waste & $0.49 \pm 2.4$ & $18,21,23,26,28$ \\
\hline & Gas/diesel & $9.12 \pm 1.72$ & 18 \\
\hline \multirow{5}{*}{$\begin{array}{l}\text { Biomass } \\
\text { burning }\end{array}$} & Forest fires & $0.88 \pm 1.68$ & $18,21,23,26,28$ \\
\hline & Deforestation fires & $0.88 \pm 1.68$ & $18,21,23,26,28$ \\
\hline & Peat fires & $0.59 \pm 2.02$ & $18,21,23,28$ \\
\hline & Woodland fires & $0.88 \pm 1.68$ & $18,21,23,26,28$ \\
\hline & Savanna fires & $0.59 \pm 2.02$ & $18,21,23,28$ \\
\hline \multirow{7}{*}{$\begin{array}{l}\text { International } \\
\text { shipping }\end{array}$} & Ocean tanker & $48.98 \pm 1.25$ & $29-39$ \\
\hline & Ocean container ships & $48.98 \pm 1.25$ & $29-39$ \\
\hline & Ocean bulk and combined carriers & $48.98 \pm 1.25$ & $29-39$ \\
\hline & General cargo vessels & $48.98 \pm 1.25$ & $29-39$ \\
\hline & Non-cargo vessels & $48.98 \pm 1.25$ & $29-39$ \\
\hline & Auxiliary engines & $48.98 \pm 1.25$ & $29-39$ \\
\hline & Military vessels & $48.98 \pm 1.25$ & $29-39$ \\
\hline
\end{tabular}

$a$. EFs were directly obtained from our previous study considering both international fuel trade and FGD control measures ${ }^{17}$.

b. EFs were calculated by using sulfur content (SC) and ash content (AC) (see Table S3) using the equation: $E F=S C \times(1-A C) \times(1-\gamma)$, where the sulfur control rate from FGD were directly from our previous study ${ }^{17}$.

c. EFs were directly obtained from our previous study considering both international fuel trade and sulfur removal during petroleum refinery ${ }^{17}$.

d. Unit of EF is $\mathrm{kg} \mathrm{S} / \mathrm{kg}$ product, which were used to calculate EFs regarding no control measures.

$e$. EFs were calculated using linear regression models (see Table S4). 
Table S3 List of sulfur content (SC in \%) and ash content (AC in \%) in lignite.

\begin{tabular}{|c|c|c|c|}
\hline Country $^{a}$ & SC & $\mathbf{A C}$ & Ref. \\
\hline Russia & 0.25 & 22.3 & 40 \\
\hline Germany & 1 & 7.19 & 41 \\
\hline Czech Republic & 2.42 & 20.73 & 42 \\
\hline China & 0.95 & 20.13 & 43 \\
\hline Canada & 0.33 & 14.17 & 44 \\
\hline Hungary & 7.03 & 27.37 & 45 \\
\hline Greece & 1.64 & 38.36 & 46 \\
\hline Turkey & 3.3 & 21.49 & 47 \\
\hline Poland & 3 & 15.37 & 48 \\
\hline Romania & 2.45 & 3.34 & 49 \\
\hline Bulgaria & 2.64 & 19.1 & 50 \\
\hline Australia & 2.2 & 9.42 & 51,52 \\
\hline United States & 1.6 & 11.9 & 53 \\
\hline
\end{tabular}

a. $\mathrm{SC}=1.80 \%$ and $\mathrm{AC}=15.22 \%$ were adopted to all the other countries following Spiro et al., $1992^{28}$.

Table S4 Regression models of EFs for motor vehicles. The regression model can be described as: $\log (E F)=\operatorname{alog}\left(G D P_{c a p}\right)+b$, where $G D P_{c a p}$ represents per-capita GDP for a given country.

\begin{tabular}{llllll}
\hline Source & $\mathbf{N}$ & $\mathbf{R}^{\mathbf{2}}$ & $\boldsymbol{a}$ & $\boldsymbol{b}$ & Ref. \\
\hline Vehicle(gasoline) & 67 & 0.56 & -1.63 & 12 & $11,18,22,54-63$ \\
Vehicle(diesel) & 58 & 0.45 & -1.35 & 12 & $11,18,22,54-63$ \\
\hline
\end{tabular}


Table 55 Comparisons of global anthropogenic $\mathrm{SO}_{2}$ emissions between this study and previous literatures in the latest years.

\begin{tabular}{|c|c|c|c|}
\hline Data source & Emission year & $\mathrm{SO}_{2}$ emissions, $\mathrm{Tg}$ & This study \\
\hline EDGAR4.3.2 ${ }^{8}$ & 2012 & 102.9 & $107.0(97.1 \sim 121.7)$ \\
\hline EDGAR4.2 $2^{64}$ & 2008 & 125.5 & $110.6(100.1 \sim 125.6)$ \\
\hline HTAP2. $2^{65}$ & 2010 & 106.9 & $109.2(99.0 \sim 124.3)$ \\
\hline CEDS $^{9}$ & 2014 & 111.7 & $105.4(95.8 \sim 119.8)$ \\
\hline HYDE $^{66}$ & 2000 & 150.3 & $103.1(92.8 \sim 117.1)$ \\
\hline Smith et al., $2011^{19}$ & 2005 & 115.5 & $108.1(97.7 \sim 122.6)$ \\
\hline Klimont et al., $2013^{67}$ & 2011 & 101.0 & $109.7(99.7 \sim 124.8)$ \\
\hline Lamarque et al., $2010^{68}$ & 2000 & 92.7 & $103.1(92.8 \sim 117.1)$ \\
\hline Lee et al., $2011^{69}$ & 2006 & 112.9 & $111.0(100.3 \sim 125.9)$ \\
\hline
\end{tabular}

Table S6 Comparisons of turning point of Environmental Kuznets Curve (EKC) for $\mathrm{SO}_{2}$ emissions between this study and previous ones.

\begin{tabular}{lllll}
\hline Country/Region & Study period & $\begin{array}{l}\text { Emission data } \\
\text { source }\end{array}$ & $\begin{array}{l}\text { Turning point } \\
\text { (USD 2010 constant) }\end{array}$ & References \\
\hline World & $1960-1990$ & ASL & 170000 & 70 \\
World & $1984-2000$ & Stern2005 & 506785 & 71 \\
World & $1970-2005$ & EPI2012 & 24300 & 72 \\
World & 2014 & & 30000 & This study \\
OECD countries & $1970-1992$ & OECD & 11570 & 73 \\
OECD countries & $1960-1990$ & ASL & 15492 & 70 \\
OECD countries & $1984-2000$ & Stern2005 & 16445 & 71 \\
\hline OECD countries & 2014 & & 16034 & This study \\
\hline Non-OECD countries & $1960-1990$ & ASL & 1522870 & 70 \\
Non-OECD countries & $1984-2000$ & Stern2005 & No tuning point & 71 \\
\hline Non-OECD countries & 2014 & & 78624 & This study \\
\hline
\end{tabular}




\section{References}

(1) Li, C.; McLinden, C.; Fioletov, V.; Krotkov, N.; Carn, S.; Joiner, J.; Streets, D.; He, H.; Ren, X. R.; Li, Z. Q.; Dickerson, R. R. India Is Overtaking China as the World's Largest Emitter of Anthropogenic Sulfur Dioxide. Sci. Rep. 2017, 7, 14304.

(2) Koukouli, M. E.; Theys, N.; Ding, J.; Zyrichidou, I.; Mijling, B.; Balis, D.; Ronalad van der, A. Updated SO ${ }_{2}$ emission estimates over China using OMI/Aura observations. Atmos. Meas. Tech. 2018, 11 (3), 1817-1832.

(3) Liu, F.; Choi, S.; Li, C.; Fioletov, V. E.; McLinden, C. A.; Joiner, J.; Krotkov, N. A.; Bian, H.; JanssensMaenhout, G.; Darmenov, A. S.; da Silva, A. M. A new global anthropogenic $\mathrm{SO}_{2}$ emission inventory for the last decade: a mosaic of satellite-derived and bottom-up emissions. Atmos. Chem. Phys. 2018, 18, 1657116586.

(4) Qu, Z.; Henze, D. K.; Theys, N.; Wang, J.; Wang, W. Hybrid mass balance/4D-Var joint inversion of NOx and SO2 emissions in East Asia. J. Geophys. Res. Atmos. 2019, 124 (14), 8203-8224.

(5) Qu, Z.; Henze, D. K.; Li, C.; Theys, N.; Wang, Y.; Wang, J.; Wang, W.; Han, J.; Shim, C.; Dickerson, R. R.; Ren, X. SO $\mathrm{SO}_{2}$ emission estimates using $\mathrm{OMI} \mathrm{SO}_{2}$ retrievals for 2005-2017. J. Geophys. Res. Atmos. 2019, 124 (14), 8336-8359.

(6) Miyazaki, K.; Sekiya, T.; Fu, D.; Bowman, K. W.; Kulawik, S. S.; Sudo, K.; Walker, T.; Kanaya, Y.; Takigawa, M.; Ogochi, K.; Eskes, H. Balance of emission and dynamical controls on ozone during the Korea-United States Air Quality campaign from multiconstituent satellite data assimilation. J. Geophys. Res. Atmos. 2019, 124 (1), 387-413.

(7) Li, M.; Klimont, Z.; Zhang, Q.; Martin, R. V.; Zheng, B.; Heyes, C.; Cofala, J.; Zhang, Y.; He, K. Comparison and evaluation of anthropogenic emissions of $\mathrm{SO}_{2}$ and $\mathrm{NO}_{2}$ over China. Atmos. Chem. Phys. 2018, 18, 3343-3456.

(8) Crippa, M.; Guizzardi, D.; Muntean, M.; Schaaf, E.; Dentener, F.; van Aardenne, J. A.; Monni, S.; Doering, U.; Olivier, J. G. J.; Pagliari, V.; Janssens-Maenhout, G. Gridded emissions of air pollutants for the period 1970-2012 within EDGAR v4.3.2. Earth Syst. Sci. Data 2018, 10 (4), 1987-2013.

(9) Hoesly, R. M.; Smith, S. J.; Feng, L. Y.; Klimont, Z.; Janssens-Maenhout, G.; Pitkanen, T.; Seibert, J. J.; Vu, L.; Andres, R. J.; Bolt, R. M.; Bond, T. C.; Dawidowski, L.; Kholod, N.; Kurokawa, J.; Li, M.; Liu, L.; Lu, Z. F.; Moura, M. C. P.; O'Rourke, P. R.; Zhang, Q. Historical (1750-2014) anthropogenic emissions of reactive gases and aerosols from the Community Emissions Data System (CEDS). Geosci. Model Dev. 2018, 11 (1), 369-408.

(10) Stohl, A.; Aamaas, B.; Amann, M.; Baker, L. H.; Bellouin, N.; Berntsen, T. K.; Boucher, O.; Cherian, R.; Collins, W.; Daskalakis, N.; Dusinska, M.; Eckhardt, S.; Fuglestvedt, J. S.; Harju, M.; Heyes, C.; Hodnebrog, O.; Hao, J.; Im, U.; Kanakidou, M.; Klimont, Z.; Kupiainen, K.; Law, K. S.; Lund, M. T.; Maas, R.; MacIntosh, C. R.; Myhre, G.; Myriokefalitakis, S.; Olivie, D.; Quaas, J.; Quennehen, B.; Raut, J. C.; Rumbold, S. T.; Samset, B. H.; Schulz, M.; Seland, O.; Shine, K. P.; Skeie, R. B.; Wang, S.; Yttri, K. E.; Zhu, T. Evaluating the climate and air quality impacts of short-lived pollutants. Atmos. Chem. Phys. 2015, 15 (18), 10529-10566.

(11) Xing, J.; Pleim, J.; Mathur, R.; Pouliot, G.; Hogrefe, C.; Gan, C. M.; Wei, C. Historical gaseous and primary aerosol emissions in the United States from 1990 to 2010. Atmos. Chem. Phys. 2013, 13 (15), 7531-7549.

(12) United States Environmental Protection Agency, National Emissions Inventory (NEI) Data, https://www.epa.gov/air-emissions-inventories/national-emissions-inventory-nei (accessed 2019.6)

(13) National emissions reported to the Convention on Long-range Transboundary Air Pollution (LRTAP Convention), https://www.eea.europa.eu/data-and-maps/data/national-emissions-reported-to-the-conventionon-long-range-transboundary-air-pollution-lrtap-convention-13 (accessed 2019.10). 
(14) Zheng, B.; Tong, D.; Li, M.; Liu, F.; Hong, C.; Geng, G.; Li, H.; Li, X.; Peng, L.; Qi, J.; Yan, L.; Zhang, Y.; Zhao, H.; Zheng, Y.; He, K.; Zhang, Q. Trends in China's anthropogenic emissions since 2010 as the consequence of clean air actions. Atmos. Chem. Phys. 2018, 18 (19), 14095-14111.

(15) National bureau of statistics of China. China Statistical Yearbook. China Statistics Press, Beijing.

(16) Lu, Z.; Zhang, Q.; Streets, D. G. Sulfur dioxide and primary carbonaceous aerosol emissions in China and India, 1996-2010. Atmos. Chem. Phys. 2011, 11 (18), 9839-9864.

(17) Zhong, Q.; Shen, H.; Yun, X.; Chen, Y.; Ren, Y. a.; Xu, H.; Shen, G.; Ma, J.; Tao, S. Effects of International Fuel Trade on Global Sulfur Dioxide Emissions. Environ. Sci. Tech. Let. 2019, 6 (12), 727-731.

(18) Greenhouse Gas and Air Pollution Interactions and Synergies (GAINS), International Institute for Applied Systems Analysis (IIASA), http://www.iiasa.ac.at/web/home/research/researchPrograms/Global emissions.html (accessed 2019.4).

(19) Smith, S. J.; van Aardenne, J.; Klimont, Z.; Andres, R. J.; Volke, A.; Arias, S. D. Anthropogenic sulfur dioxide emissions: 1850-2005. Atmos. Chem. Phys. 2011, 11 (3), 1101-1116.

(20) Arndt, R. L.; Carmichael, G. R.; Streets, D. G.; Bhatti, N. Sulfur dioxide emissions and sectorial contributions to sulfur deposition in Asia. Atmos. Environ. 1997, 31 (10), 1553-1572.

(21) Venkataraman, C.; Chandramouli, B.; Patwardhan, A. Anthropogenic sulphate aerosol from India: estimates of burden and direct radiative forcing. Atmos. Environ. 1999, 33 (19), 3225-3235.

(22) Garg, A.; Shukla, P. R.; Bhattacharya, S.; Dadhwal, V. K. Sub-region (district) and sector level $\mathrm{SO}_{2}$ and NOx emissions for India: assessment of inventories and mitigation flexibility. Atmos. Environ. 2001, 35 (4), 703-713.

(23) Andreae, M. O.; Merlet, P. Emission of trace gases and aerosols from biomass burning. Global Biogeochem. Cy. 2001, 15 (4), 955-966.

(24) Smith, S. J.; Pitcher, H.; Wigley, T. M. L. Global and regional anthropogenic sulfur dioxide emissions. Global Planet. Change 2001, 29 (1-2), 99-119.

(25) Smith, K. R. Air-Pollution - Assessing Total Exposure in Developing-Countries. Environment 1988, 30 (10), 17.

(26) Kato, N. Analysis of structure of energy consumption and dynamics of emissions of atmospheric species related to the global environmental change ( $\mathrm{SOx}, \mathrm{NOx}$, and $\mathrm{CO}_{2}$ ) in Asia. Atmos. Environ 1996, 30 (5), 757 785.

(27) Streets, D. G.; Waldhoff, S. T. Biofuel use in Asia and acidifying emissions. Energy 1998, 23 (12), 10291042.

(28) Spiro, P. A.; Jacob, D. J.; Logan, J. A. Global inventory of sulfur emissions with $1^{\circ} \times 1^{\circ}$ resolution. $J$. Geophys. Res. Atmos. 1992, 97 (D5), 6023-6036.

(29) Murphy, S. M.; Agrawal, H.; Sorooshian, A.; Padro, L. T.; Gates, H.; Hersey, S.; Welch, W. A.; Jung, H.; Miller, J. W.; Cocker, D. R.; Nenes, A.; Jonsson, H. H.; Flagan, R. C.; Seinfeld, J. H. Comprehensive Simultaneous Shipboard and Airborne Characterization of Exhaust from a Modern Container Ship at Sea. Environ. Sci. Technol. 2009, 43 (13), 4626-4640.

(30) Chen, G.; Huey, L. G.; Trainer, M.; Nicks, D.; Corbett, J.; Ryerson, T.; Parrish, D.; Neuman, J. A.; Nowak, J.; Tanner, D.; Holloway, J.; Brock, C.; Crawford, J.; Olson, J. R.; Sullivan, A.; Weber, R.; Schauffler, S.; Donnelly, S.; Atlas, E.; Roberts, J.; Flocke, F.; Hubler, G.; Fehsenfeld, F. An investigation of the chemistry of ship emission plumes during ITCT 2002. J. Geophys. Res. 2005, 110, D10.

(31) Sinha, P.; Hobbs, P. V.; Yokelson, R. J.; Christian, T. J.; Kirchstetter, T. W.; Bruintjes, R. Emissions of trace gases and particles from two ships in the southern Atlantic Ocean. Atmos. Environ. 2003, 37 (15), 21392148 . 
(32) Hobbs, P. V.; Garrett, T. J.; Ferek, R. J.; Strader, S. R.; Hegg, D. A.; Frick, G. M.; Hoppel, W. A.; Gasparovic, R. F.; Russell, L. M.; Johnson, D. W.; O’Dowd, C.; Durkee, P. A.; Nielsen, K. E.; Innis, G. Emissions from ships with respect to their effects on clouds. J. Atmos. Sci. 2000, 57 (16), 2570-2590.

(33) Eyring, V.; Köhler, H. W.; van Aardenne, J.; Lauer, A. Emissions from international shipping: the last 50 years. J. Geophys. Res. 2005, 110, D17305.

(34) Agrawal, H.; Welch, W. A.; Miller, J. W.; Cockert, D. R. Emission measurements from a crude oil tanker at sea. Environ. Sci. Technol. 2008, 42 (19), 7098-7103.

(35) Corbett, J. J.; Winebrake, J. J.; Green, E. H.; Kasibhatla, P.; Eyring, V.; Lauer, A. Mortality from ship emissions: A global assessment. Environ. Sci. Technol. 2007, 41 (24), 8512-8518.

(36) Corbett, J.J.; Kohler, H.W. Updated emissions from ocean shipping. J. Geophys. Res. 2003, 108, D20.

(37) Endresen, Ø.; Sørgård, E.; Sundet, J. K.; Dalsøren, S. B.; Isaksen, I. S. A.; Berglen, T. F.; Gravir, G. Emission from international sea transportation and environmental impact. J. Geophys. Res. 2003, 108 (D17), 4560.

(38) Endresen, Ø.; Sørgard, E.; Behrens, H. L.; Brett, P. O.; Isaksen, I. S. A. A historical reconstruction of ships' fuel consumption and emissions. J. Geophys. Res. 2007, 112, D12301.

(39) Khan, M. Y.; Giordano, M.; Gutierrez, J.; Welch, W. A.; Asa-Awuku, A.; Miller, J. W.; Cocker, D. R. Benefits of two mitigation strategies for container vessels: cleaner engines and cleaner fuels. Environ. Sci. Technol. 2012, 46 (9), 5049-5056.

(40) Kouprianov, V. I. Influence of lignite quality on airborne emissions from power generation in the Russian Far East and in Northern Thailand. Fuel Process Technol. 2002, 76 (3), 187-199.

(41) Oleschko, H.; Schimrosczyk, A.; Lippert, H.; Muller, M. Influence of coal composition on the release of Na-, $\mathrm{K}-, \mathrm{Cl}$-, and S-species during the combustion of brown coal. Fuel 2007, 86 (15), 2275-2282.

(42) Bouska, V.; Pesek, J. Quality parameters of lignite of the North Bohemian Basin in the Czech Republic in comparison with the world average lignite. Int. J. Coal. Geol. 1999, 40 (2-3), 211-235.

(43) Chen, B.; Wei, X.-Y.; Zong, Z.-M.; Yang, Z.-S.; Qing, Y.; Liu, C. Difference in chemical composition of supercritical methanolysis products between two lignites. Appl. Energ. 2011, 88 (12), 4570-4576.

(44) Goodarzi, F.; Hower, J. C. Classification of carbon in Canadian fly ashes and their implications in the capture of mercury. Fuel 2008, 87 (10-11), 1949-1957.

(45) Hámor-Vidó, M.; Hámor, T. Sulphur and carbon isotopic composition of power supply coals in the Pannonian Basin, Hungary. Int. J. Coal. Geol. 2007, 71 (4), 425-447.

(46) Koukouzas, N.; Hämäläinen, J.; Papanikolaou, D.; Tourunen, A.; Jäntti, T. Mineralogical and elemental composition of fly ash from pilot scale fluidised bed combustion of lignite, bituminous coal, wood chips and their blends. Fuel 2007, 86 (14), 2186-2193.

(47) Küçükbayrak, S.; Ersoy-Herigboyu, A.; Haykin-Açma, H.; Guner, H.; Urkan, K. Investigation of the relation between chemical composition and ash fusion temperatures for soke Turkish lignites. Fuel Sci. Technol. Int. 1993, 11 (9), 1231-1249.

(48) Lorenc-Grabowska, E.; Gryglewicz, G. Adsorption of lignite-derived humic acids on coal-based mesoporous activated carbons. J. Colloid Interface Sci. 2005, 284 (2), 416-23.

(49) Peuravuori, J.; Žbánková, P.; Pihlaja, K. Aspects of structural features in lignite and lignite humic acids. Fuel Process Technol. 2006, 87 (9), 829-839.

(50) Stefanova, M.; Oros, D. R.; Otto, A.; Simoneit, B. R. Polar aromatic biomarkers in the Miocene Maritza-East lignite, Bulgaria. Organic Geochemistry 2002, 33 (9), 1079-1091.

(51) Ward, C. R.; Li, Z.; Gurba, L. W. Variations in elemental composition of macerals with vitrinite reflectance and organic sulphur in the Greta Coal Measures, New South Wales, Australia. Int. J. Coal. Geol. 2007, 69 
(3), 205-219.

(52) Yani, S.; Zhang, D. An experimental study into pyrite transformation during pyrolysis of Australian lignite samples. Fuel 2010, 89 (7), 1700-1708.

(53) Winschel, R. A. The relationship of carbon dioxide emissions with coal rank and sulfur content. J. Air Waste Manage. 1990, 40 (6), 861-865.

(54) Chiang, H. L.; Hwu, C. S.; Chen, S. Y.; Wu, M. C.; Ma, S. Y.; Huang, Y. S. Emission factors and characteristics of criteria pollutants and volatile organic compounds (VOCs) in a freeway tunnel study. Sci Total Environ 2007, 381 (1-3), 200-211.

(55) Wang, X.; Westerdahl, D.; Wu, Y.; Pan, X.; Zhang, K. M. On-road emission factor distributions of individual diesel vehicles in and around Beijing, China. Atmos. Environ. 2011, 45 (2), 503-513.

(56) Westerdahl, D.; Wang, X.; Pan, X.; Zhang, K. M. Characterization of on-road vehicle emission factors and microenvironmental air quality in Beijing, China. Atmos. Environ. 2009, 43 (3), 697-705.

(57) Hueglin, C.; Buchmann, B.; Weber, R. O. Long-term observation of real-world road traffic emission factors on a motorway in Switzerland. Atmos. Environ. 2006, 40 (20), 3696-3709.

(58) Zhang, Y. L.; Wang, X. M.; Li, G. H.; Yang, W. Q.; Huang, Z. H.; Zhang, Z.; Huang, X. Y.; Deng, W.; Liu, T. Y.; Huang, Z. Z.; Zhang, Z. Y. Emission factors of fine particles, carbonaceous aerosols and traces gases from road vehicles: Recent tests in an urban tunnel in the Pearl River Delta, China. Atmos. Environ. 2015, $122,876-884$.

(59) Shah, I. H.; Zeeshan, M. Estimation of light duty vehicle emissions in Islamabad and climate co-benefits of improved emission standards implementation. Atmos. Environ. 2016, 127, 236-243.

(60) Mishra, D.; Goyal, P. Estimation of vehicular emissions using dynamic emission factors: A case study of Delhi, India. Atmos. Environ. 2014, 98, 1-7.

(61) Baidya, S.; Borken-Kleefeld, J. Atmospheric emissions from road transportation in India. Energ. Policy 2009, 37 (10), 3812-3822.

(62) Herndon, S. C.; Shorter, J. H.; Zahniser, M. S.; Wormhoudt, J.; Nelson, D. D.; Demerjian, K. L.; Kolb, C. E. Real-time measurements of $\mathrm{SO}_{2}, \mathrm{H}_{2} \mathrm{CO}$, and $\mathrm{CH}_{4}$ emissions from in-use curbside passenger buses in New York City using a chase vehicle. Environ. Sci. Technol. 2005, 39(20), 7984-7990.

(63) Chang, S. C.; Lin, T. H.; Lee, C. T. On-road emission factors from light-duty vehicles measured in Hsuehshan Tunnel (12.9 km), the longest tunnel in Asia. Environ. Monit. Assess. 2009, 153 (1-4), 187-200.

(64) European Commission, Joint Research Centre (JRC)/Netherlands Environmental Assessment Agency (PBL). Emission Database for Global Atmospheric Research, release version 4.2 (EDGAR4.2) https://edgar.jrc.ec.europa.eu/overview.php?v=42 (accessed 2015.12).

(65) Janssens-Maenhout, G.; Crippa, M.; Guizzardi, D.; Dentener, F.; Muntean, M.; Pouliot, G.; Keating, T.; Zhang, Q.; Kurokawa, J.; Wankmuller, R.; van der Gon, H. D.; Kuenen, J. J. P.; Klimont, Z.; Frost, G.; Darras, S.; Koffi, B.; Li, M. HTAP_v2.2: a mosaic of regional and global emission grid maps for 2008 and 2010 to study hemispheric transport of air pollution. Atmos. Chem. Phys. 2015, 15 (19), 11411-11432.

(66) Van Aardenne, J. A.; Dentener, F. J.; Olivier, J. G. J.; Klein Goldewijk, C. G. M.; Lelieveld. J. A High Resolution Dataset of Historical Anthropogenic Trace Gas Emissions for the Period 1890-1990. Global Biogeochem. Cy. 2001, 15 (4), 909-928.

(67) Klimont, Z.; Smith, S. J.; Cofala, J. The last decade of global anthropogenic sulfur dioxide: 2000-2011 emissions. Environ. Res. Lett. 2013, 8 (1).

(68) Lamarque, J. F.; Bond, T. C.; Eyring, V.; Granier, C.; Heil, A.; Klimont, Z.; Lee, D.; Liousse, C.; Mieville, A.; Owen, B.; Schultz, M. G.; Shindell, D.; Smith, S. J.; Stehfest, E.; Van Aardenne, J.; Cooper, O. R.; Kainuma, M.; Mahowald, N.; McConnell, J. R.; Naik, V.; Riahi, K.; van Vuuren, D. P. Historical (1850- 
2000) gridded anthropogenic and biomass burning emissions of reactive gases and aerosols: methodology and application. Atmos. Chem. Phys. 2010, 10 (15), 7017-7039.

(69) Lee, C.; Martin, R. V.; van Donkelaar, A.; Lee, H.; Dickerson, R. R.; Hains, J. C.; Krotkov, N.; Richter, A.; Vinnikov, K.; Schwab, J. J. SO2 emissions and lifetimes: Estimates from inverse modeling using in situ and global, space-based (SCIAMACHY and OMI) observations. J Geophys Res-Atmos 2011, 116.

(70) Stern, D. I.; Common, M. S. Is there an environmental Kuznets curve for sulfur? J. Environ. Econ. Manag. 2001, 41 (2), 162-178

(71) Cole, M. Re-examining the pollution-income relationship: a random coefficients approach. Economic Bulletin 2005, 14 (1), 1-7.

(72) Halkos, G.; Managi, S. Recent advances in empirical analysis on growth and environment: introduction. Environ. Dev. Econ. 2017, 22 (6), 649-657.

(73) Cole, M. A.; Rayner, A.J.; Bates, J.M. The environmental Kuznets curve: an empirical analysis. Environ. Dev. Econ. 1997, 2, 401-416. 